OPEN ACCESS

Edited by:

Vanesa Esteban,

Instituto de Investigación Sanitaria de

la Fundación Jiménez Díaz, Spain

Reviewed by:

Michael Breitenbach,

University of Salzburg, Austria

Sinisa Savic,

University of Leeds, United Kingdom

${ }^{*}$ Correspondence:

Mariana Castells

mcastells@bwh.harvard.edu

Specialty section: This article was submitted to Inflammation,

a section of the journal

Frontiers in Immunology

Received: 31 May 2017 Accepted: 20 October 2017 Published: 08 November 2017

Citation:

Castells M (2017) Drug Hypersensitivity and Anaphylaxis in Cancer and Chronic Inflammatory

Diseases: The Role of

Desensitizations.

Front. Immunol. 8:1472. doi: 10.3389/fimmu.2017.01472

\section{Drug Hypersensitivity and Anaphylaxis in Cancer and Chronic Inflammatory Diseases: The Role of Desensitizations}

\section{Mariana Castells* \\ Allergy and Immunology, Brigham and Women's Hospital, Harvard Medical School, Boston, MA, United States}

Drug allergy is a rising problem in the twenty-first century which affects all populations and races, children, and adults, and for which the recognition, diagnosis, management, and treatment is still not well standardized. Classical and new chemotherapy drugs, monoclonal antibodies (MoAbs), and small molecules to treat cancer and chronic inflammatory diseases are aimed at improving quality of life and life expectancy of patients, but an increasing number of reactions including anaphylaxis precludes their use in targeted populations. Women are more affected by drug allergy and up to $27 \%$ of women with ovarian and breast cancer develop carboplatin allergy after multiple cycles of treatment. Carriers of BRCA genes develop drug allergy after fewer exposures and can present with severe reactions, including anaphylaxis. Atopic patients are at increased risk for chemotherapy and MoAbs drug allergy and the current patterns of treatment with recurrent and intermittent drug exposures may favor the development of drug allergies. To overcome drug allergy, desensitization has been developed, a novel approach which provides a unique opportunity to protect against anaphylaxis and to improve clinical outcomes. There is evidence that inhibitory mechanisms blocking IgE/antigen mast cell activation are active during desensitization, enhancing safety. Whether desensitization modulates drug allergic and anaphylactic responses facilitating tolerance is currently being investigated. This review provides insight into the current knowledge of drug allergy and anaphylaxis to cancer and chronic inflammatory diseases drugs, the mechanisms of drug desensitization and its applications to personalized medicine.

Keywords: desensitization, monoclonal antibodies in cancer, platins, drug allergy, taxanes

\section{INTRODUCTION}

With the unprecedented use of chemotherapies drugs and targeted monoclonal antibodies (MoAbs) and small molecules in the twenty-first century, increased hypersensitivity reactions (HSRs) have emerged worldwide $(1,2)$. Drug allergic reactions are unexpected, can be severe including anaphylaxis and prevent the use of first-line therapies, with consequent impact in patient's survival and quality of life $(3,4)$. These reactions range from mild cutaneous manifestations such as pruritus and hives to life-threatening anaphylaxis with hypotension, oxygen desaturation and cardiovascular collapse, and deaths have been reported after re-exposure to allergic drugs $(5,6)$. The presentation of symptoms can be atypical such as pain, which has been associated with taxenes reactions, and chills and fever which have been seen with oxaliplatin and MoAbs reactions $(7,8)$. Delayed reactions occurring 
more than $24 \mathrm{~h}$ after chemotherapy infusions can be due to the prolonged half-life of MoAbs and the presence of premedications, which may mask the acute phase of the reactions $(9,10)$.

The traditional classification of drug hypersensitivity and allergy into the classical types I-IV does not encompass the current spectrum of reactions and symptoms occurring in cancer patients and patients with chronic inflammatory diseases $(11,12)$. Some of the reactions have no known underlying mechanism, others have a known mechanism which is not part of the four described types and some drugs can induce mixed reactions with two or more proposed mechanisms $(13,14)$. Hypersensitivity to rituximab, a chimeric anti-CD20 MoAb, can induce cytokine-like reactions with chills, fevers, hypotension, and oxygen desaturation, which have been attributed to the release of cytokines such as IL-6, IL-1 $\beta$, and TNF- $\alpha$ and are named cytokine release syndrome or cytokine storm, which is not contemplated in the Gell and Coombs classification (15). In contrast, some patients have classical IgE-mediated reactions to rituximab and have presented positive skin testing demonstrating that IgE and mast cells are part of the underlying mechanism (16). Some patients reactive to oxaliplatin present mixed reactions with Type I features such as hives and hypotension, along with fever and chills as seen in cytokine storm-like reactions, presenting a complex mixed pattern of reactivity which provides challenges to management and treatment (17). During mixed reactions tryptase, the major mast cell protease, and IL- 6 can be elevated in serum indicating mast cell activation and cytokine release from unknown cellular sources. Reactions to taxenes can trigger direct mast cell/basophil activation with elevation of serum tryptase with or without evidence of IgE, indicating that more than one mechanism can explain taxane hypersensitivity. A different receptor than FceRI, such as the recently described MrgprX2 for drugs with THIQ motifs such as quinolones and paralyzing agents such as atracuronium could be activated during non-IgE taxane reactions $(13,18-20)$.

Patients presenting with delayed cutaneous reactions are at a great concern for Stevens-Johnson syndrome and toxic epidermal necrolysis, two life-threatening conditions which can lead to permanent disability, blindness, and dramatic decrease in the quality of life for survivors $(16,21)$. The underlying mechanisms of the reactions are poorly understood and up to now no predictive markers have been available. Genetic susceptibility and defined HLA haplotypes are thought to be risk factors for some of the reactions, such as HLA-B 5701 in HIV patients reactive to abacavir. In patients with targeted haplotypes, a new role for viral reactivation of HHV6 and other virus have been demonstrated, and the pathogenic role of the virus is under study (22-24).

To provide an operational classification which can adapt to the increasing knowledge of the mechanisms of reactions and to the symptoms and clinical presentations, a recent initiative has provided a new terminology, applicable to precision medicine. In the new categorization drug allergy phenotypes are defined by the underlying endotypes and associated biomarkers and can be used in personalized medicine, with each patient being categorized according to her/his symptoms complex presentation. Current phenotypes include acute and delayed reactions with $\operatorname{IgE}$ and
non-IgE involvement, cytokine storm, and mixed patterns. The endotypes responsible for the expression of symptoms include mast cell and basophil activation through known receptors (FceRI, FcgR, MRGPRX2) and directly through known receptors: complement, kinin and bradykinin activation and COX-1 inhibition. Associated biomarkers include serum tryptase, skin testing, basophil activation test, specific $\operatorname{IgE}$ and patch testing among others $(13,25-28)$.

Patients presenting with reactions compatible with phenotypes consistent with acute and delayed IgE and non-IgE, mast cell/basophil activation, and T cell activation endoptypes may be prevented from the use of first-line therapies for fear of inducing anaphylaxis or more severe delayed reactions upon re-exposure to the allergenic drug. A groundbreaking procedure, desensitization, has emerged in the last 15 years as a proven effective and safe procedure to maintain patients on their first-line medications.

\section{CLINICAL VIGNETTE}

Mrs. MFF is a 49-year-old healthy female who was discovered to have ovarian cancer after a routine gynecology ultrasound and was initially treated with surgery and chemotherapy with six courses of carboplatin and paclitaxel and entered remission. Two years later, the CA125 is increased and new masses are found in her abdomen, a diagnosis of recurrent stage 4 ovarian cancer is made and carboplatin and paclitaxel restarted. After the second course of carboplatin, the patient feels her hands itchy but finished the infusion and did not have any further symptoms. On the day of her third infusion, the patient presented flushing, generalized pruritus, shortness of breath, and sudden dizziness. The blood pressure drops below normal range as well as the oxygen saturation and the patient has a syncopal episode and needs to be resuscitated with epinephrine, fluids, anti-histamines, and steroids. She recovers and her diagnosis is of anaphylaxis, a serum tryptase level during the episode is elevated at $52 \mathrm{ng} / \mathrm{ml}$ (normal range $11.4 \mathrm{ng} / \mathrm{ml}$ ). The patient is evaluated for carboplatin allergy and skin testing is positive. Her options are to change to a second-line agent which is likely to reduce her life expectancy or to remain on first-line therapy with carboplatin but because of her anaphylactic reaction this option is not considered safe unless carboplatin can be introduced through desensitization, a powerful and novel intervention which has shown to protect patients against anaphylaxis and permit re-introduction of allergy drugs.

\section{DRUG DESENSITIZATION}

The term drug desensitization is currently used to define a process by which a patient's immune response to a drug is modified to generate temporary tolerance, taking advantage of well characterized inhibitory pathways (6). In the case of IgE-mediated drug allergy, positive skin testing and specific serum IgE can be used as biomarkers along with elevated serum tryptase level during the acute reaction $(8,11,17)$. Patients without evidence of $\operatorname{IgE}$ mechanism are good candidates for desensitization provided the phenotype of the drug reaction is a type I or a type IV like reaction without features of SJS/TEN $(16,21,29)$ (Table 1). 
TABLE 1 | Indications, contraindications and risk factors for drug desensitization.

\begin{tabular}{|c|c|c|}
\hline Indications & High-risk patients & Contraindications \\
\hline $\begin{array}{l}\text { Reactions type I (mast } \\
\text { cells/lgE/basofills) } \\
\text { Reaction type IV (except } \\
\text { SCARs) }\end{array}$ & $\begin{array}{l}\text { Severe anaphylaxis } \\
\text { (intubation) }\end{array}$ & $\begin{array}{l}\text { Severe cutaneous adverse } \\
\text { reactions (SCARs) (SJS/TEN } \\
\text { DIHS/DRESS, AGEP) }\end{array}$ \\
\hline No alternative drug & $\begin{array}{l}\text { Severe respiratory } \\
\text { disease }\end{array}$ & $\begin{array}{l}\text { Immunocytotoxic reactions } \\
\text { (type II reactions) }\end{array}$ \\
\hline $\begin{array}{l}\text { Drug is more effective } \\
\text { and/or associated with } \\
\text { less side effects }\end{array}$ & $\begin{array}{l}\text { Severe cardiac } \\
\text { disease }\end{array}$ & Vasculitis \\
\hline $\begin{array}{l}\text { Drug has a unique } \\
\text { mechanism of action }\end{array}$ & $\begin{array}{l}\text { Severe systemic } \\
\text { diseases } \\
\text { Use of beta-blockers, } \\
\text { ACE inhibitors } \\
\text { Pregnancy }\end{array}$ & $\begin{array}{l}\text { Serum sickness-like (type III } \\
\text { reactions) }\end{array}$ \\
\hline
\end{tabular}

SJS, Stevens-Johnson syndrome; TEN, toxic epidermal necrolysis; DIHS, drug-induced hypersensitivity syndrome; DRESS, drug reaction (rash) with eosinophilia and systemic symptoms; AGEP, acute generalized exanthematous.
The mechanisms underlying drug desensitization are based on in vitro and in vivo models which have proposed that mast cells and basophils can be induced to predominantly inhibitory pathways by small incremental antigen doses, deactivating signal transduction and mediators release (13) (Figure 1).

Negative skin test is seen following desensitization in patients with IgE-mediated reactions, providing evidence of the powerful mechanisms which turn off skin mast cells (30-32). Partitioning of an optimal dose into 11-16 incremental doses starting at 1/1,000 the target dose and delivering them with sufficient time interval to mast cells; inhibits the acute release of beta-hexosaminidase, a mast cell granule mediator, prevents the generation of arachidonic acid and products such as leukotrienes and prostaglandins and the late generation of inflammatory cytokines (Figure 1) (13).

During desensitization, calcium influx is abolished and actin polymerization impaired, providing stability to intracellular granules in an antigen-specific fashion (Figure 1) (13). Membrane events that prevent internalization or modify its

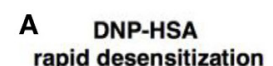

rapid desensitization

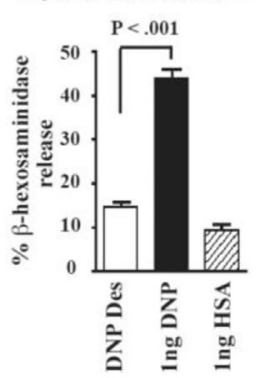

E

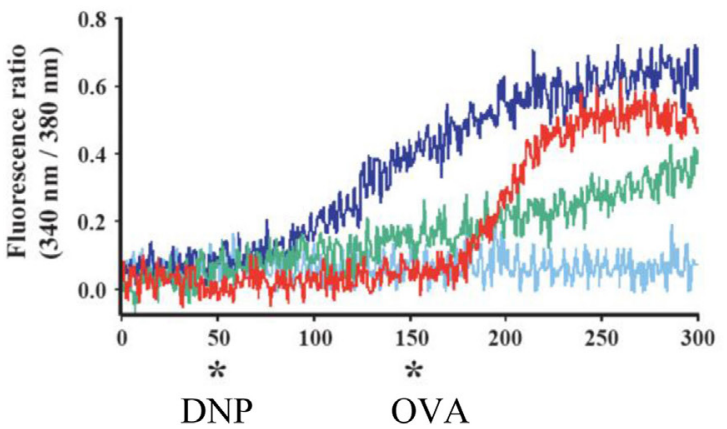

B OVA

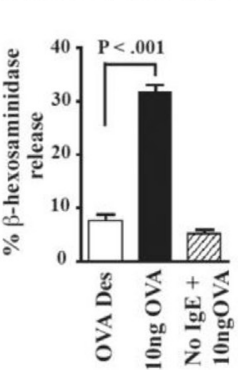

C

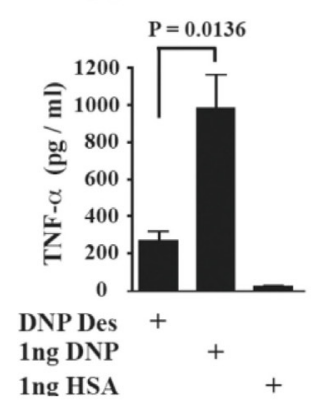

$\mathbf{F}$

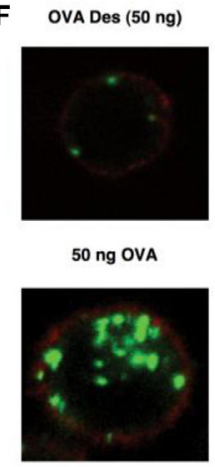

- ova-Aksa Fluor $488 \quad$ DNP-DVLight 649
D

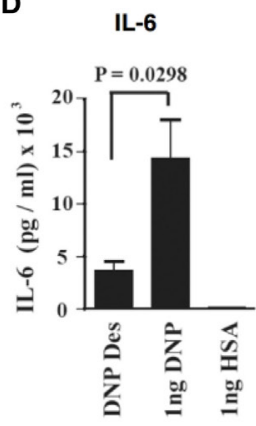

OVA Des + 10 ng DNP

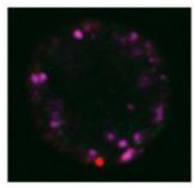

$10 \mathrm{ng}$ DNP

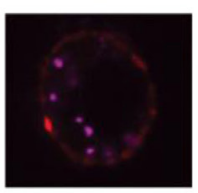

- Cholera Tosil subunil B-Alesa Flor 598

FIGURE 1 | In vitro IgE/antigen mouse mast cells activation and desensitization (13). (A) Desensitization of in vitro DNP-IgE sensitized mouse mast cells with DNP inhibits the release of granule mediators such as beta-hexosaminidase. Instead of one single optimal dose, 11 suboptimal sequential doses are given until reaching the optimal dose. (B) Desensitization of in vitro OVA-IgE sensitized mouse mast cells with OVA inhibits the release of granule mediators such as beta-hexosaminidase. Instead of one single optimal dose, 11 suboptimal sequential doses are given until reaching the optimal dose. (C) Desensitization of in vitro DNP-IgE sensitized mouse mast cell mediators with DNP inhibits the de novo generation of cytokines TNF alpha and IL-6 (D). (E) Calcium entry (blue line) occurs after activation of DNP-IgE sensitized mouse mast cells with DNP but not when cells have been desensitized to DNP (red line, * DNP). Desensitization is specific since cells that were DNP desensitized and OVA-IgE sensitized presented calcium entry after OVA activation (red line, * OVA). (F) Membrane events are modified during desensitization with lack of internalization of desensitized antigens: left panels indicate that OVA desensitization does not present internalization of antigen (upper panel) as opposed to activation (lower panel with green labeled OVA internalized) and right panels indicate that after OVA desensitization another non desensitizing antigen such as DNP can be internalized (upper panel) as seen with activation (lower panel). 


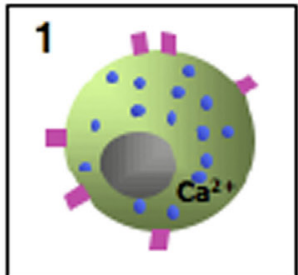

Resting mast cell

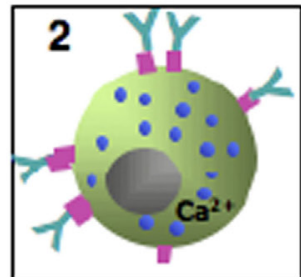

Sensitizaticn

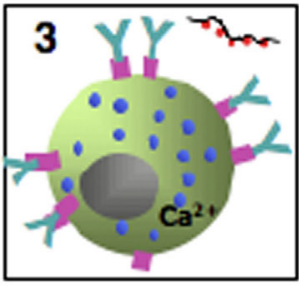

Antigen addition

\section{ACTIVATION}

(one antigen/lgE/FCeRI complex is shown)
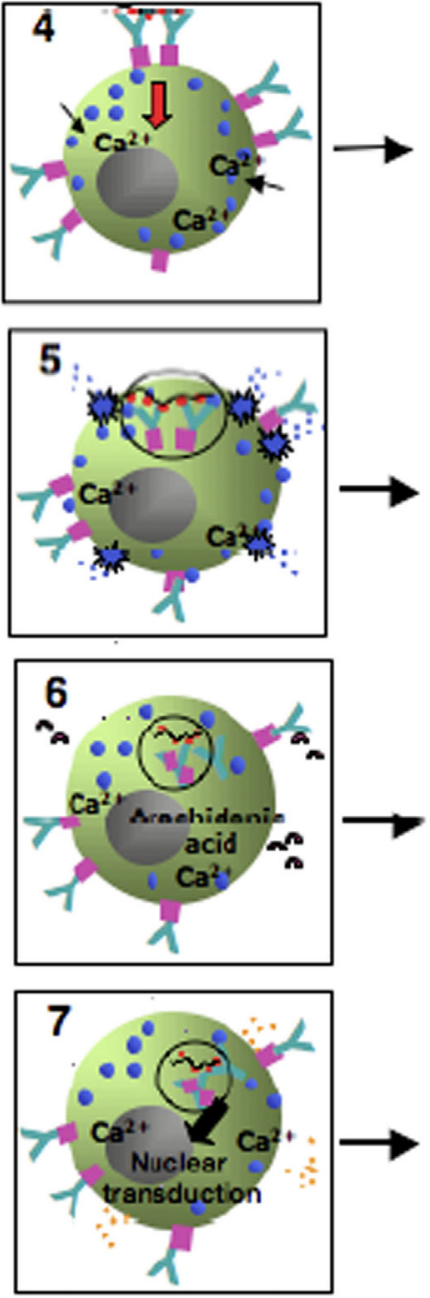
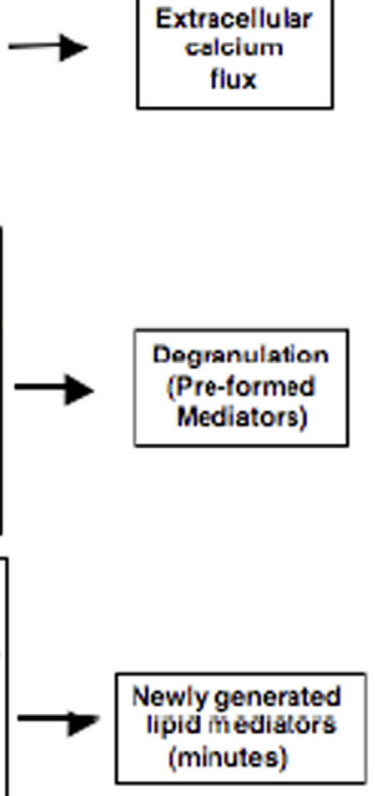

Degranulation (Pre-formed Mediators)
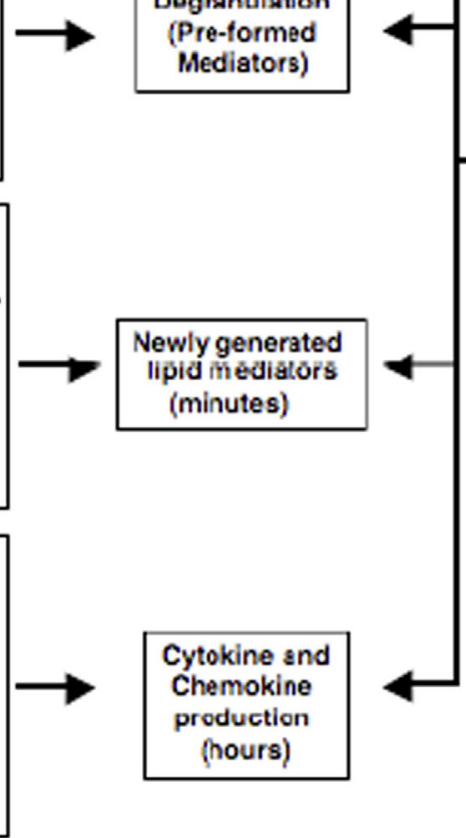

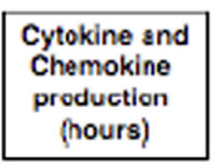

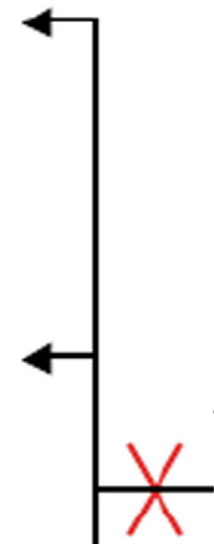

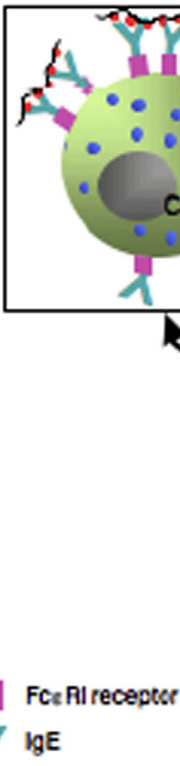

Auttivalont Antigen

\section{DESENSITIZATION}

(11 step protocol)
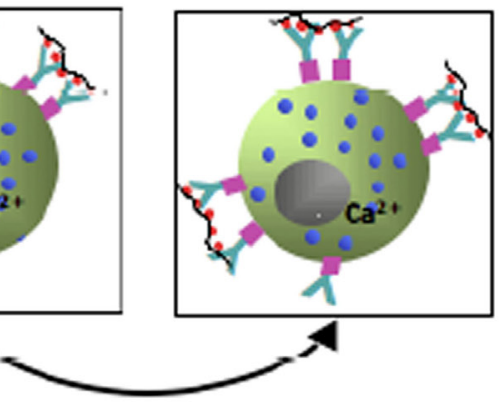

Membrane

re-arrangement

of receptors

- Preformed Mediaxors

A Nevly generated ipld mediators

Cylokines and Cremokines

FIGURE 2 | Model of in vitro mouse mast cells activation and desensitization. The left side cartoons provide the steps of antigen/lgE/FceRI activation starting from antigen cross-linking, internalization, calcium entry and release of granule mediators, generation of lipd mediators such as prostaglandins and leukotrienes, and production of late phase cytokines. The right sided panel provides the hypothetical membrane capping and rearrangement occurring during the delivery of sequential suboptimal doses of allergen in desensitization preventing internalization of antigen, calcium entry, and mediators release. 


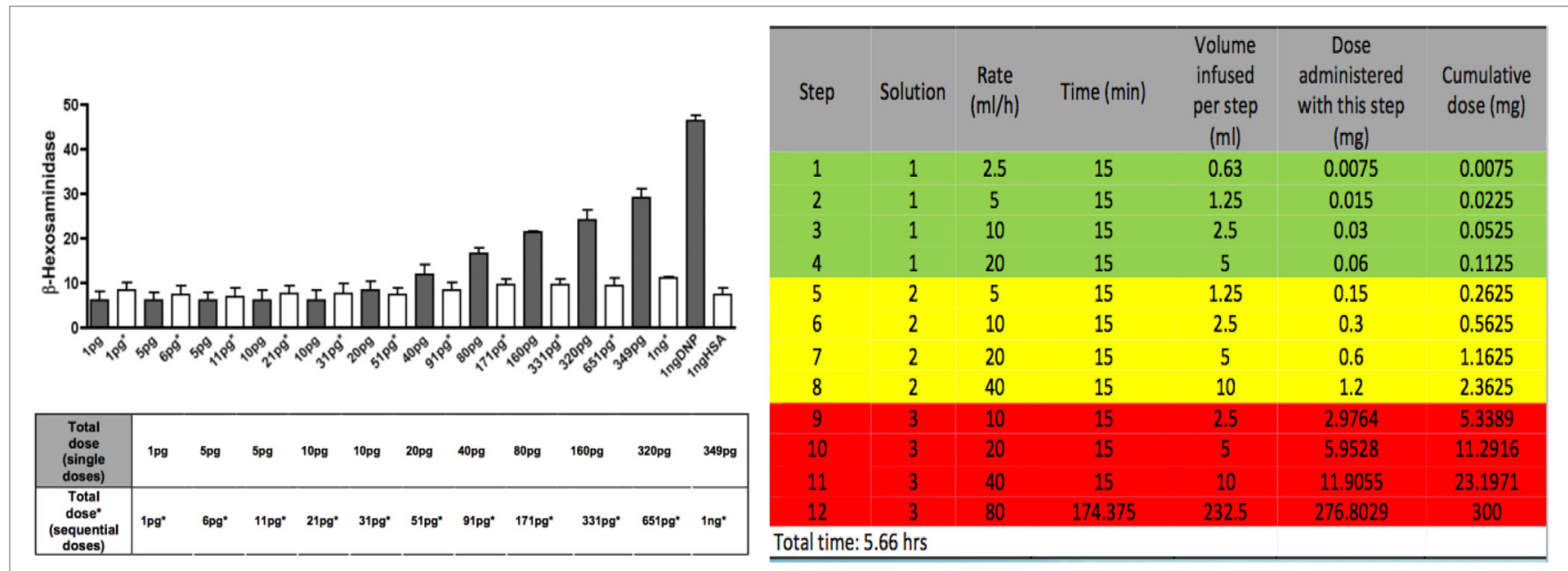

FIGURE 3 | In vitro and in vivo protocols for induction of IgE/antigen desensitization. In the left panel in black, increasing single doses of antigen induce a dose response release of beta-hexosaminidase in vitro mouse mast cells. In white, the same doses given sequentially induce a profound inhibition of the beta -hexosaminidase release. In the right panel, a model of desensitization protocol used for human desensitization in which 3 bags and 12 steps (4 steps per bag) are used to administer sequential doubling doses every $15 \mathrm{~min}$ which provides the target dose of $300 \mathrm{mg}$ after $5.66 \mathrm{~h}$ when the last step is completed.

response to subthreshold doses of antigen occur during desensitization and are associated with incremental unresponsiveness to specific antigen (Figure 2) (13). Association of the FceRI to ITIM containing receptors, capable of dephosphorylating ITAMs receptors has been postulated as one of the mechanisms of desensitization. Mast cells desensitized to one antigen are responsive to a second non desensitizing antigen, providing evidence of compartimentalization and highly specialized and regulated intracellular processes (Figure 1) (13).

The protocols used for in vitro desensitization have been adapted in vivo and further adaptations have produced safe protocols for human use (Figure 3) with similar dose increments and interval times (25-28). These human protocols have now been used in thousands of cases with remarkable safety since the inhibitory mechanisms of desensitization protect against anaphylaxis (Figure 4) $(33,34)$.

Whether after multiple desensitizations neutralizing antibodies can be generated which may block allergenic drug epitopes has been hypothesized (6). Maintaining drug desensitization state depends upon continued drug exposure and desensitized drugs require administration at regular intervals to maintain a stable pharmacokinetics state. Desensitization needs to be repeated if several half-lives of the medication have elapsed $(6,33)$.

Protocols for drug desensitization have been successfully used for antibiotics, chemotherapy drugs, and MoAbs among other drugs in patients with IgE and non-IgE-mediated HSRs (Figure 5) $(6,14,33,35)$. The phenotypes of reactions amendable to desensitization include immediate and delayed reactions. Typical type I reactions usually begin within minutes of initiation of the infusion to few hours after the infusion due to anti-histamine and steroid premedication. The signs and symptoms include puritus, flushing, urticaria, angioedema, throat tightening, wheezing, nausea, diarrhea, hypotension, syncope, seizures, and cardiovascular collapse which can lead to death. Atypical symptoms include back,

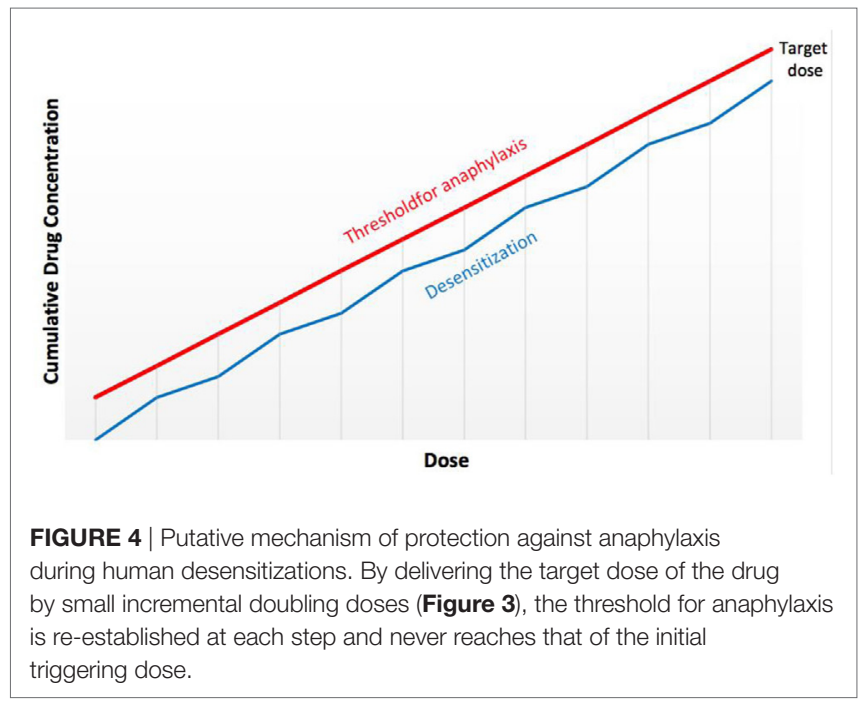

chest, or abdominal pain (such as seen with taxanes, oxaliplatin, MoAbs such as rituximab) $(33,35)$.

Delayed reactions are attributed to type IV reactions and can occur several days after the infusion and are typically limited to the skin with maculopapular rashes (36). Reactions that involve mucosal membranes and/or are associated with systemic symptoms are not amenable to desensitization due to the risk of inducing a severe systemic reaction with small amounts of drug antigen $(16,21,29)$.

Desensitization should be considered in patients with reaction phenotypes consistent with type I and type IV reactions who have no alternative therapy or for whom alternative therapies are of less value or can induce more side effects. The algorithm for the evaluation of these patients is seen in Figure $\mathbf{6}(6,34,37)$. The 


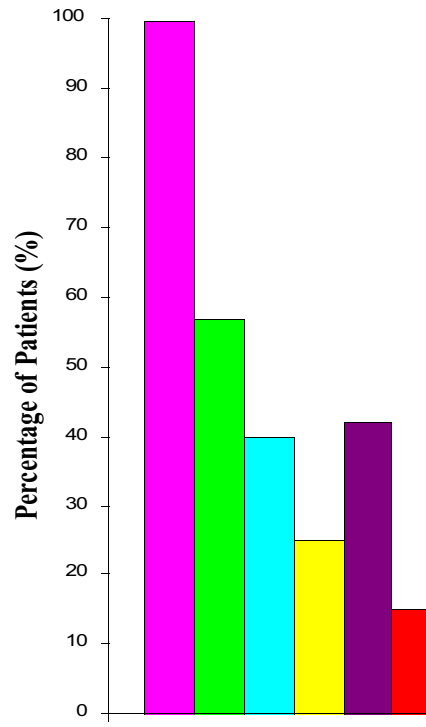

Carboplatin

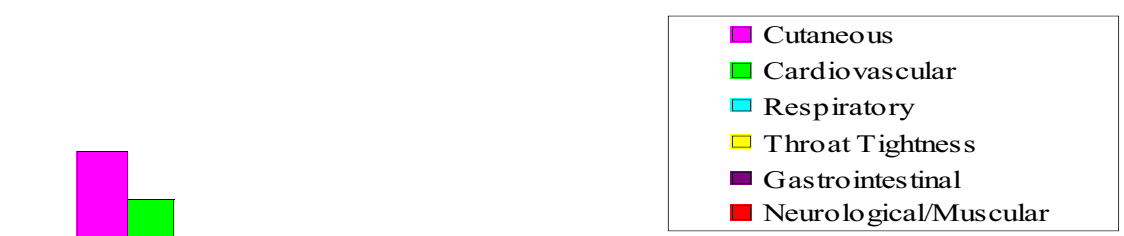

URE 5 Symptoms and signs of hypersensitivity reactions amendable to desensitization. Carboplatin and other paltins such as cisplatin and oxaliplatin reactions include classical symptoms of anaphylaxis with cutaneous, respiratory, cardiovascular, and gastrointestinal symptoms. Reactions to taxenes including paclitaxel and docetaxel present with pain as a neuromuscular symptoms in up to $4 \%$ of the patients. Doxorubicin/adriamycin and other chemotherapies present with sudden onset hypo or hypertension in up to $60 \%$ of patients and rituximab and other monoclonal antibodies present with cutaneous and cardiovascular symptoms in $70 \%$ of the patients.

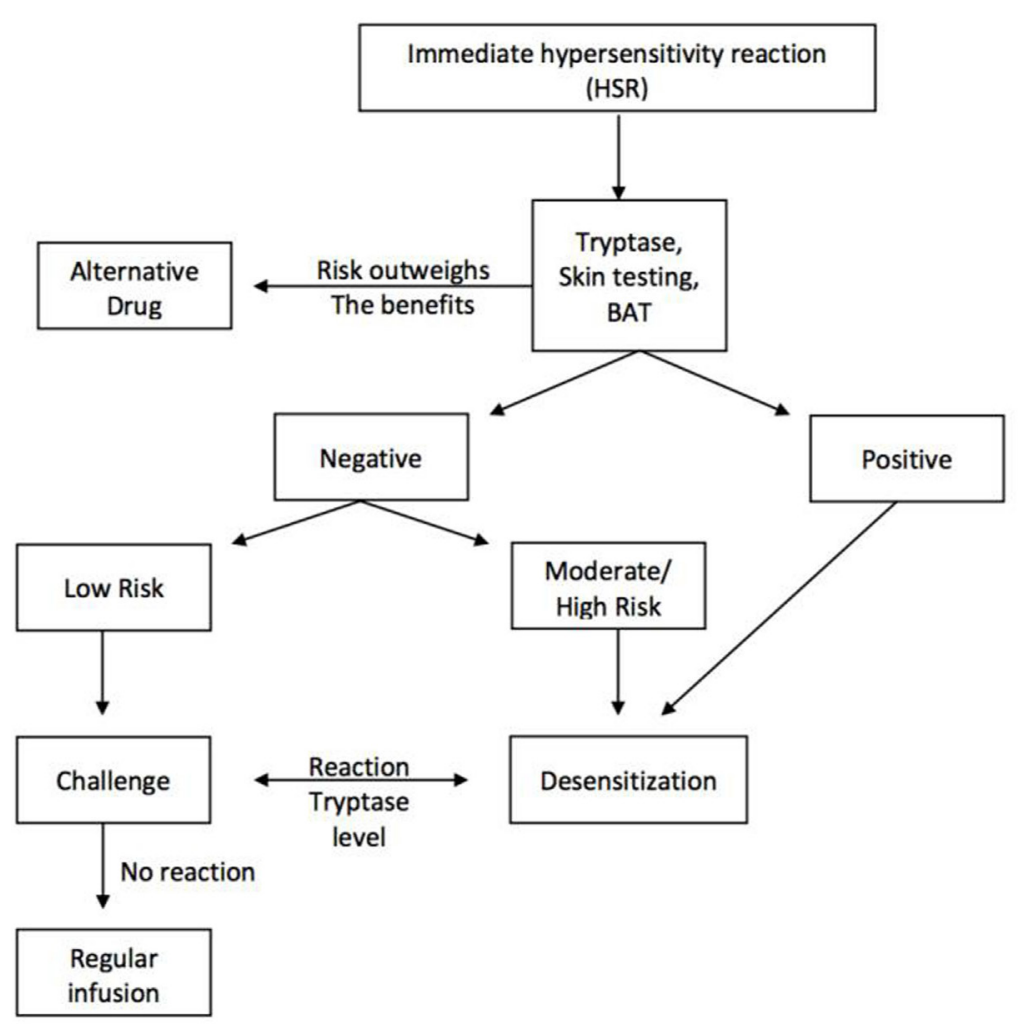

FIGURE 6 | Algorithm for the evaluation of drug hypersensitivity reactions and the role of desensitization for the re-introduction of the first-line medications, when no alternative is available or the alternative does not provide the same benefits or life expectancy as the first line. 
nature and symptoms of the initial reactions needs to be established and tryptase and skin test provide evidence of IgE and/ or mast cell involvement. BAT is a research tool and cannot be applied to current clinical practice.

Drug desensitization can be performed in patients of any age and in pregnant women when alternative therapies are not possible or when delaying therapy may incur a shortened life span. Anaphylaxis is the major risk during desensitization since patients are exposed to their allergic drug. Large series have demonstrated that most breakthrough reactions during desensitization are mild and less severe than the patient's initial HSR and fatalities have not been reported, but all desensitizations can only be performed by an expert allergist familiar with the personalized protocol and potential reactions (Table 1) $(17,33,35)$. The safety of desensitizations is paramount and patients with grade 3 severe initial reactions and anaphylaxis can be desensitized with minimal reactions as seen in Table 2.

Desensitization is not recommended for type II and type III serum sickness-like reactions or in patients with reactions with skin desquamation, EM, Stevens-Johnson syndrome or toxic epidermal necrolysis, because small amounts of drug can induce irreversible and potentially fatal reactions (Table 1) $(16,21,29)$.

The most commonly used intravenous desensitization protocols are standardized 12- to 16-step protocols modeled after in vitro protocols and can be personalized to all drugs with adjustment of the target dose, time intervals between doses and starting dose (Figure 3) $(33,38)$. Protocols are available for intravenous desensitizations but also for oral, subcutaneous, intraperitoneal, or intravenous routes in the outpatient and inpatient settings (39-41). Desensitization for delayed reactions is also available and may take several days but recent data suggest that some of these reactions may be amenable to shorter time intervals $(8,36,42)$. The overall safety of desensitizations is similar for all medications provided the mechanism of the initial reaction is of type I, IgE and non-IgE or type IV. As seen in Figure 7, the overall safety indicates that $93 \%$ of patients present with no reaction or grade 1 reactions and all completed the desensitization.

Platins, taxanes, and MoAbs are the most common chemotherapy currently used in desensitization and are described below.

\section{Platin Hypersensitivity}

Platinum compounds are used in ovarian, colorectal, endometrial, glioblastoma, lung, and pancreatic cancer as initial chemotherapy

TABLE 2 | Safety of first desensitization in patients with grades 1, 2, and 3 initial reactions.

\begin{tabular}{lcccrr}
\hline & \multicolumn{5}{c}{ First desensitization reaction grade } \\
\cline { 2 - 6 } $\begin{array}{l}\text { Initial } \\
\text { hypersensitivity } \\
\text { reaction grade }\end{array}$ & $\mathbf{0}$ & $\mathbf{1}$ & $\mathbf{2}$ & $\mathbf{3}$ & Total \\
\hline 1 & & & & & \\
2 & $76(61 \%)$ & $38(30 \%)$ & $7(6 \%)$ & $4(3 \%)$ & 125 \\
3 & $38(58 \%)$ & $22(34 \%)$ & $4(6 \%)$ & $1(2 \%)$ & 65 \\
Total & $122(60 \%)$ & $54(26 \%)$ & $10(5 \%)$ & $19(9 \%)$ & 205 \\
\hline
\end{tabular}

From Sloane et al. (33). and in second-line or salvage settings. Carboplatin is the most popular since it is less nephrotoxic and neurotoxic than cisplatin. Allergic reactions to platins are IgE-mediated and require sensitization through multiple exposures, with $27 \%$ of women becoming allergic after seven life time exposures $(12,43,44)$. Allergic symptoms typically start at the second round of treatment, when the cancer recurs and after 1-2 exposures sensitized patients present with flushing and pruritus which can progress to shortness of breath with further exposure and can lead to anaphylaxis, with hypotension and cardiovascular collapse $(2,4$, $7,33,45)$. Patients bearing BRCA 1 and 2 gene mutations have an increased risk for carboplatin reactions, which can occur with fewer exposures $(46,47)$. Most reactions to platins occur during or shortly after the drug infusion and the phenotype is that of type I reaction. In a study of 60 carboplatin sensitized patients, $100 \%$ had cutaneous, $60 \%$ pulmonary, $40 \%$ respiratory, and $42 \%$ gastrointestinal symptoms (6).

The phenotype of reactions to oxaliplatin can be more complex with features including typical IgE-mediated symptoms and atypical symptoms such as back and pelvic pain and cytokinemediated fever and chills $(7,11,17,48,49)$. Antibody-mediated thrombocytopenia and immune complex-mediated syndromes with urticaria and proteinuria have also been observed $(17,50)$.

Skin testing to platins has been safely done (Table 3) and is diagnostic tool to demonstrate an IgE/mast cell mechanism in patients with carboplatin and cisplatin reactions $(7,11,17)$. For patients exposed to six or more courses of carboplatin in the last 6 months the positive predictive value is up to $86 \%$ (11). Oxaliplatin skin testing is negative in up to $50 \%$ of patients presenting type I reactions, indicating other than IgE mechanisms or lack of skin test allergenic determinants (17). Circulating serum specific IgE has been demonstrated and patients reactive to oxaliplatin with detectable serum-specific IgE have also demonstrated IgE to carboplatin and cisplatin without exposure, indicating broad cross-reactivity (51). IgE to platins can be short lived since a study has demonstrated that ST is negative in a high proportion of patients with a remote history of HSR to carboplatin, but re-exposure leads to resensitization and severe reactions (11). When platins are considered as first-line therapy, desensitization is a safe option since increased premedications alone do not prevent anaphylaxis and cross-reactivity may prevent the use of other platins (51). Patients with severe cutanoues reactions, SJS and TEN are currently not candidates since the mechanism of the reactions is unknown and small amounts of medications may induce severe symptoms (1, 16, 21, 29). Desensitization provides a similar life expectancy as non-allergic non desensitized patients (Figure 8) without increased health costs (33).

\section{Taxanes}

Taxanes are used in gynecologic, lung, breast, and prostate cancers and reactions to taxenes are among the most frequent chemotherapy reactions and fatalities have been reported (52). Paclitaxel and docetaxel have been the more frequently used and more recently other taxenes such as cabacitaxel and abraxene have become popular (53). Paclitaxel is an insoluble compound originally isolated from the bark of the pacific yew tree, Taxus 


\section{A}

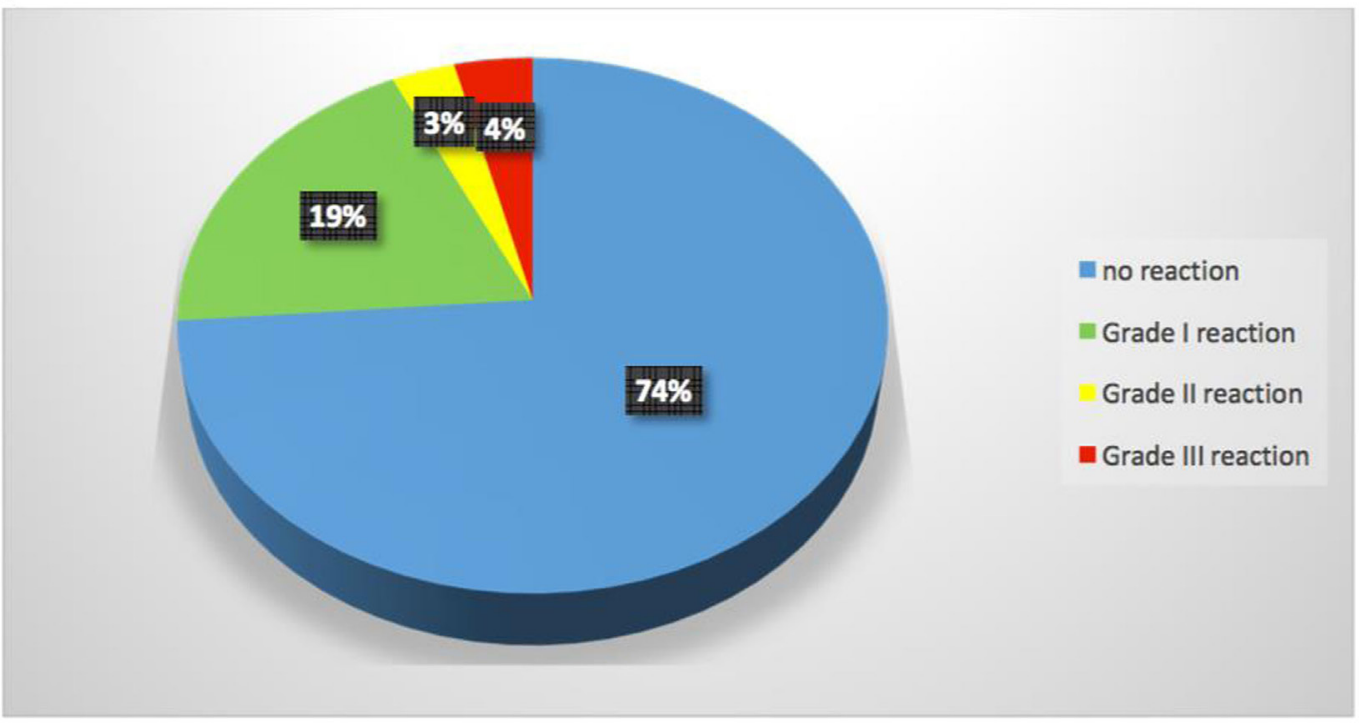

B

C

D

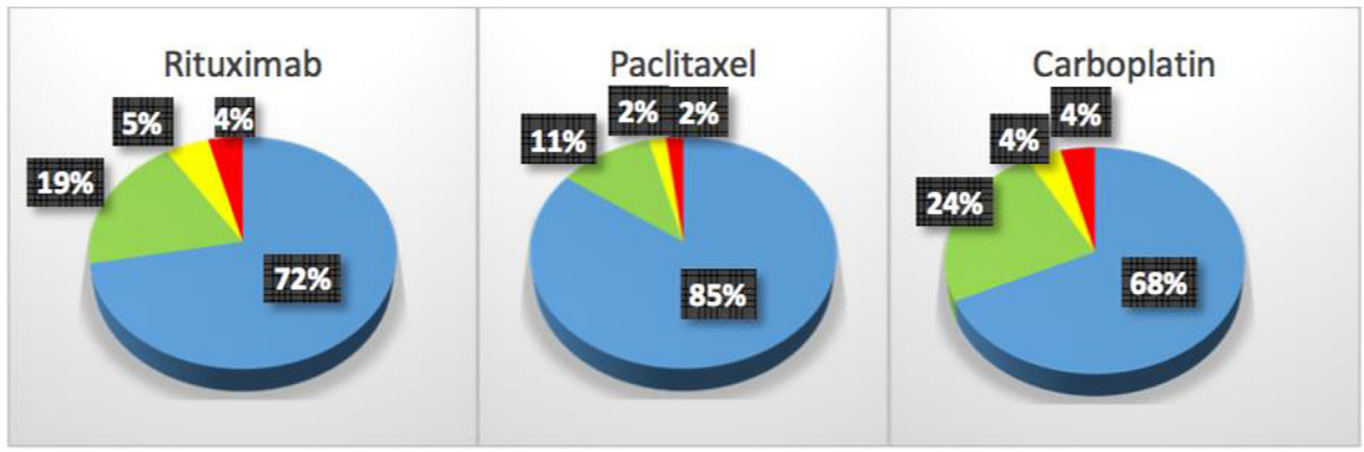

FIGURE 7 | The overall safety of desensitization for common chemotherapy drugs and monoclonal antibodies.

TABLE 3 | Skin testing for the diagnosis of chemotherapy drug allergy including platins, monoclonal antibodies, and paclitaxel.

\begin{tabular}{lcc}
\hline Medication & Prick $\mathbf{( m g / m l )}$ & Intradermal $\mathbf{( m g / m l )}$ \\
\hline Carboplatin & 10 & $0.1,1,5$, and 10 \\
Cisplatin & 1 & 0.1 and 1 \\
Oxaliplatin & 5 & 0.5 and 5 \\
Rituximab & 10 & $0.1,1$, and 10 \\
Infliximab & 10 & $0.1,1$, and 10 \\
Tocilizumab & 20 & $0.2,2$, and 20 \\
Centuximab & 20 & $0.2,2$, and 20 \\
Traztuzmab & 21 & $0.21,2.1$, and 21 \\
Bevacizumab & 25 & $0.25,2.5$, and 25 \\
Cyclophosphamide & 10 & $0.1,1$, and 10 \\
Methotrexate & 25 & $0.2,2.5$, and 25 \\
Paclitaxel & $1-6$ & 0.001 and 0.01 \\
\hline
\end{tabular}

Baccata tree and solubilized in cremophor and docetaxel is a semi-synthetic molecule derived from a precursor found in European yew tree needles and solubilized in polysorbate 80 $(36,54)$. The solvents can cause complement activation, generating anaphylotoxins $\mathrm{C} 3 \mathrm{a}$ and $\mathrm{C} 5 \mathrm{a}$ and leading to mast cell activation (55-57). Taxanes are used with premedications including anti-histamines and steroids due to a high rate of reactions in early clinical studies (54). The rate of reaction has decreased to less than $10 \%$ and typically occurs during the first or second lifetime exposure in up to $80 \%$ of the patients (54). The phenotype of the reactions include type I symptoms such as throat tightness, flushing, hypotension, and dyspnea but atypical symptoms such as chest, back, or pelvic pain $(8,36,54)$.

Skin testing has uncovered IgE-mediated reactions to taxanes and a recent study reported that 103 of 145 taxane reactive patients (71\%) had positive results. Negative skin test patients who were challenged were likely to tolerated taxane infusions without desensitization. Atopy was present in over $40 \%$ of the patients and because patients react at first or second exposure suggested prior sensitization or cross-reactivity with environmental allergens (8). Risk stratification based on biomarkers such as skin testing can safely guide the management to taxane reactions and allows a significant number of patients to resume regular infusions. For patients with positive skin test and significant initial reaction for whom taxanes are first-line therapy, desensitization should be considered (8). 


\section{Monoclonal Antibodies}

There are over 45 MoAbs currently in use for the treatment of cancer and inflammatory and autoimmune diseases. Reactions to MoAbs depend on their structure and vary from chimeric mouse-human, humanized, to fully human. Some of the most frequently used MoAbs are presented in Table 4, including their targets, incidence of overall injection/infusion site reactions, and HSRs $(19,34,35)$.

Monoclonal antibodies immunogenicity depends on the human content but fully human MoAbs, such as adalimumab

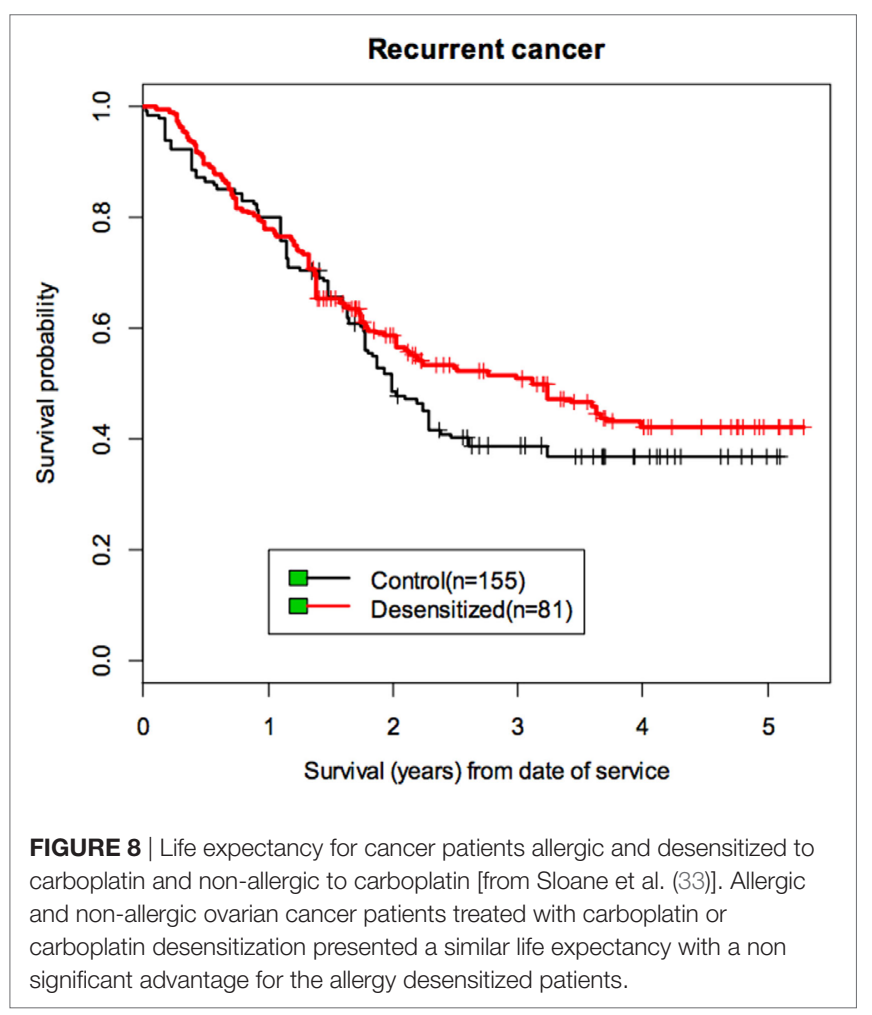

and ofatumumab can induce severe HSRs likely due to the glycosylation patterns in vitro and the generation of neo antigens (58). This is best exemplified in reactions to cituximab which can occur at first exposure in patients sensitized through tick bites to the mammalian oligosaccharide epitope, galactose-alpha1,3-galactose (alpha-gal) (59).

The phenotypes of MoAbs reactions include limited infusion reactions, IgE-mediated reactions, serum sickness-like reactions, cytokine storm-like reactions, and mixed reactions. Infusion reactions are characterized by nausea, chills, fever, and malaise and for trastuzumab these reactions can occur in up to $40 \%$ of patients $(34,35)$. Like cytokine storm-like reactions, which are more severe, can associate with hypotension, oxygen desaturation, and require treatment with steroids and COX-1 inhibitors, proinflammatory cytokines (such as IL- 6 and TNF- $\alpha$ ) are thought to be involved $(60,61)$.

Immediate and delayed HSRs can occur with MoAbs and serum sickness-like reactions, such as seen with infliximab and omalizumab, which can present with rash, myalgia, fever, polyarthralgias, pruritus, edema, and fatigue (35).

Monoclonal antibodies used subcutaneously can elicit injection-site reactions few hours after the injection and persisting for several days. The phenotype of these reactions include local redness, warmth, burning, itching, urticaria, pain, and induration, varying in frequency from 0.8 to $4.5 \%$ with certolizumab to up to $45 \%$ with omalizumab (39).

Reactions to MoAbs can occur during the infusion and should prompt interruption of the treatment and the evaluation of tryptase and inflammatory cytokines to further understand the mechanism of the reactions. Skin testing with the offending agent can be done for type I and mixed reactions 2-4 weeks after the reaction to avoid false negative results, in particular in anaphylactic reactions in which natural desensitization can occur (35). An important consideration is cost of MoAbs; there are no available reagents at the present time for the evaluation of $\mathrm{MoAb}$ reactions and using a treatment vial may exceed several thousand dollars, precluding a diagnostic skin test evaluation. The negative

TABLE 4 | Common monoclonal antibodies in use and rate of overall reactions and hypersensitivity reactions (HSR).

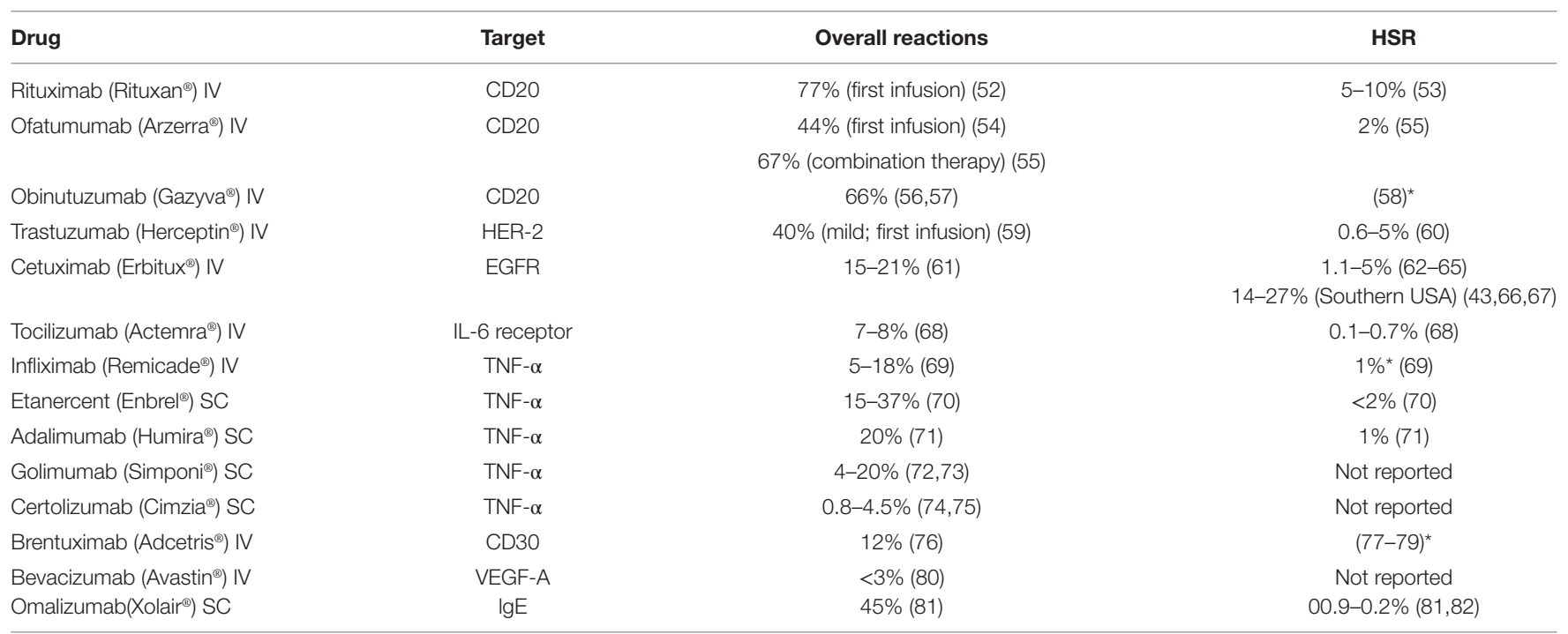

${ }^{*}<p=0.05$ 
predictive value of skin testing is not known and in a study of 23 patients reactive to trastuzumab, infliximab, or rituximab, 13 patients had positive skin test. Positive and negative skin test patient with significant initial reactions are candidates for desensitization and subcutaneous protocols are available. Successful desensitizations to rituximab, ofatumumab, obinutuzumab, trastuzumab, cetuximab, tocilizumab, infliximab, etanercept, adalimumab, golimumab, certolizumab, brentuximab, bevacizumab, and omalizumab have been reported (33-35).

\section{CONCLUSION}

Drug hypersensitivity is an increasing health hazard, which can compromise the quality of life and the life expectancy of cancer patients and patients with chronic inflammatory diseases with reactions to their first-line therapy. Recognition of the mechanisms of the reactions into phenotypes, understanding the underlying endotypes and evaluation of biomarkers is key to personalized medicine and enhanced patient safety allowing for informed decisions regarding drug re-exposure and desensitization.

\section{REFERENCES}

1. Kadoyama K, Kuwahara A, Yamamori M, Brown JB, Sakaeda T, Okuno Y. Hypersensitivity reactions to anticancer agents: data mining of the public version of the FDA adverse event reporting system, AERS. J Exp Clin Cancer Res (2011) 30:93. doi:10.1186/1756-9966-30-93

2. Sakaeda T, Kadoyama K, Yabuuchi H, Niijima S, Seki K, Shiraishi Y, et al. Platinum agent-induced hypersensitivity reactions: data mining of the public version of the FDA adverse event reporting system, AERS. Int J Med Sci (2011) 8(4):332-8. doi:10.7150/ijms.8.332

3. Nektaria M, Ekaterini S, Ioannis K, Leonidas M, Muhammad Wasif S. Hypersensitivity reactions associated with platinum antineoplastic agents: a systematic review. Met Based Drugs (2010) 2010. Available from: http:// downloads.hindawi.com/journals/mbd/2010/207084.pdf

4. Markman M. Toxicities of the platinum antineoplastic agents. Expert Opin Drug Saf (2003) 2(6):597-607. doi:10.1517/14740338.2.6.597

5. A fatal anaphylactic reaction to paclitaxel is described, which was preceded by a possible delayed reaction to the initial infusion. Allergy Asthma Proc (2011) 32(1):79. doi:10.2500/aap.2011.32.3409

6. Castells MC, Tennant NM, Sloane DE, Hsu FI, Barrett NA, Hong DI, et al. Hypersensitivity reactions to chemotherapy: outcomes and safety of rapid desensitization in 413 cases. J Allergy Clin Immunol (2008) 122(3):574-80. doi:10.1016/j.jaci.2008.02.044

7. Caiado J, Castells M. Presentation and diagnosis of hypersensitivity to platinum drugs. Curr Allergy Asthma Rep (2015) 15(4):15. doi:10.1007/ s11882-015-0515-3

8. Picard M, Pur L, Caiado J, Giavina-Bianchi P, Galvão VR, Berlin ST, et al. Risk stratification and skin testing to guide re-exposure in taxane-induced hypersensitivity reactions. J Allergy Clin Immunol (2016) 137(4):1154-64.e1-12. doi:10.1016/j.jaci.2015.10.039

9. Feldweg AM, Lee C-W, Matulonis UA, Castells M. Rapid desensitization for hypersensitivity reactions to paclitaxel and docetaxel: a new standard protocol used in 77 successful treatments. Gynecol Oncol (2005) 96(3):824-9. doi:10.1016/j.ygyno.2004.11.043

10. Cheifetz A, Mayer L. Monoclonal antibodies, immunogenicity, and associated infusion reactions. Mt Sinai J Med (2005) 72(4):250-6.

11. Wang AL, Patil SU, Long AA, Banerji A. Risk-stratification protocol for carboplatin and oxaliplatin hypersensitivity: repeat skin testing to identify drug allergy. Ann Allergy Asthma Immunol (2015) 115(5):422-8. doi:10.1016/j. anai.2015.07.017

12. Patil SU, Long AA, Ling M, Wilson MT, Hesterberg P, Wong JT, et al. A protocol for risk stratification of patients with carboplatin-induced
Serum tryptase, skin testing, BAT, and specific IgE are helpful diagnostic tools which will be complemented in the future with genotyping to identify patients at risk before reactions occur. Appropriate treatment of the reactions including epinephrine use and management with personalized desensitization protocols can enhance the quality of life, life expectancy, and safety of an increasing at risk population of patients with cancer and inflammatory diseases allergic to their best medications. Most patients with reactions with phenotypes consistent with type I and type IV reactions are candidates for desensitization, which can provide advancement of personalized treatments. Drug desensitization protects against anaphylaxis and activates inhibitory mechanisms which need further research to uncover cellular and molecular players amendable to pharmacological applications, which can make desensitization safer and more effective.

\section{AUTHOR CONTRIBUTIONS}

All authors listed have made a substantial, direct, and intellectual contribution to the work and approved it for publication.

hypersensitivity reactions. J Allergy Clin Immunol (2012) 129(2):443-7. doi:10.1016/j.jaci.2011.10.010

13. Sancho-Serra Mdel C, Simarro M, Castells M. Rapid IgE desensitization is antigen specific and impairs early and late mast cell responses targeting FceRI internalization. Eur J Immunol (2011) 41(4):1004-13. doi:10.1002/ eji.201040810

14. Liu A, Fanning L, Chong H, Fernandez J, Sloane D, Sancho-Serra M, et al. Desensitization regimens for drug allergy: state of the art in the 21st century. ClinExpAllergy (2011) 41(12):1679-89.doi:10.1111/j.1365-2222.2011.03825.x

15. Gamarra RM, McGraw SD, Drelichman VS, Maas LC. Serum sickness-like reactions in patients receiving intravenous infliximab. J Emerg Med (2006) 30(1):41-4. doi:10.1016/j.jemermed.2005.01.033

16. Phillips EJ, Chung W-H, Mockenhaupt M, Roujeau J-C, Mallal SA. Drug hypersensitivity: pharmacogenetics and clinical syndromes. J Allergy Clin Immunol (2011) 127(3 Suppl):S60-6. doi:10.1016/j.jaci.2010.11.046

17. Wong JT, Ling M, Patil S, Banerji A, Long A. Oxaliplatin hypersensitivity: evaluation, implications of skin testing, and desensitization. J Allergy Clin Immunol Pract (2014) 2(1):40-5. doi:10.1016/j.jaip.2013.08.011

18. McNeil BD, Pundir P, Meeker S, Han L, Undem BJ, Kulka M, et al. Identification of a mast-cell-specific receptor crucial for pseudo-allergic drug reactions. Nature (2015) 519(7542):237-41. doi:10.1038/nature14022

19. Muraro A, Lemanske RF, Castells M, Torres MJ, Khan D, Simon HU, et al. Precision medicine in allergic disease - food allergy, drug allergy, and anaphylaxis-PRACTALL document of the European Academy of Allergy and Clinical Immunology and the American Academy of Allergy, Asthma \& Immunology. Allergy (2017) Available from: http://onlinelibrary.wiley.com/doi/10.1111/all.13132/full

20. Garon SL, Pavlos RK, White KD, Brown NJ, Stone CA, Phillips EJ. Pharmacogenomics of off-target ADRs. Br J Clin Pharmacol (2017). Available from: http://onlinelibrary.wiley.com/doi/10.1111/bcp.13294/full

21. Bachot N, Roujeau J-C. Differential diagnosis of severe cutaneous drug eruptions. Am J Clin Dermatol (2003) 4(8):561-72. doi:10.2165/00128071200304080-00006

22. Mallal S, Phillips E, Carosi G, Molina JM, Workman C, Tomazic J, et al. HLA-B* 5701 screening for hypersensitivity to abacavir. N Engl J Med (2008) 358(6):568-79. doi:10.1056/NEJMoa0706135

23. Mallal S, Nolan D, Witt C, Masel G, Martin AM, Moore C, et al. Association between presence of HLA-B ${ }^{*} 5701$, HLA-DR7, and HLA-DQ3 and hypersensitivity to HIV-1 reverse-transcriptase inhibitor abacavir. Lancet (2002) 359(9308): 727-32. doi:10.1016/S0140-6736(02)07873-X

24. Hetherington S, Hughes AR, Mosteller M, Shortino D, Baker KL, Spreen W, et al. Genetic variations in HLA-B region and hypersensitivity reactions to abacavir. Lancet (2002) 359(9312):1121-2. doi:10.1016/S0140-6736(02)08158-8 
25. Ang WXG. Methods and Mechanisms of Mitigating Mast Cell Activation in Anaphylaxis. (2016). Available from: https://pdfs.semanticscholar. org/77e7/3874cddabe5b9423e1b25b2b4abaf4190b40.pdf

26. Ang WXG, Church AM, Kulis M, Choi HW, Burks AW, Abraham SN. Mast cell desensitization inhibits calcium flux and aberrantly remodels actin. J Clin Invest (2016) 126(11):4103-18. doi:10.1172/JCI87492

27. Oka T, Rios EJ, Tsai M, Kalesnikoff J, Galli SJ. Rapid desensitization induces internalization of antigen-specific IgE on mouse mast cells. J Allergy Clin Immunol (2013) 132(4):922-32.e1-16. doi:10.1016/j.jaci.2013.05.004

28. Morales AR, Shah N, Castells M. Antigen-IgE desensitization in signal transducer and activator of transcription 6-deficient mast cells by suboptimal doses of antigen. Ann Allergy Asthma Immunol (2005) 94(5):575-80. doi:10.1016/ S1081-1206(10)61136-2

29. Pirmohamed M, Friedmann PS, Molokhia M, Loke YK, Smith C, Phillips E, et al. Phenotype standardization for immune-mediated drug-induced skin injury. Clin Pharmacol Ther (2011) 89(6):896-901. doi:10.1038/clpt.2011.79

30. Cernadas JR, Brockow K, Romano A, Aberer W, Torres MJ, Bircher A, et al. General considerations on rapid desensitization for drug hypersensitivity - a consensus statement. Allergy (2010) 65(11):1357-66. doi:10.1111/j.1398-9995.2010.02441.x

31. Brockow K, Romano A, Blanca M, Ring J, Pichler W, Demoly P. General considerations for skin test procedures in the diagnosis of drug hypersensitivity. Allergy (2002) 57(1):45-51. doi:10.1034/j.1398-9995.2002.13027.x

32. MacGlashan DW Jr. IgE-dependent signaling as a therapeutic target for allergies. Trends Pharmacol Sci (2012) 33(9):502-9. doi:10.1016/j.tips.2012.06.002

33. Sloane D, Govindarajulu U, Harrow-Mortelliti J, Barry W, Hsu FI, Hong D, et al. Safety, costs, and efficacy of rapid drug desensitizations to chemotherapy and monoclonal antibodies. J Allergy Clin Immunol Pract (2016) 4(3):497-504. doi:10.1016/j.jaip.2015.12.019

34. Bonamichi-Santos R, Castells M. Diagnoses and management of drug hypersensitivity and anaphylaxis in cancer and chronic inflammatory diseases: reactions to taxanes and monoclonal antibodies. Clin Rev Allergy Immunol (2016) 1-11. doi:10.1007/s12016-016-8556-5

35. Brennan PJ, Rodriguez Bouza T, Hsu FI, Sloane DE, Castells MC. Hypersensitivity reactions to mAbs: 105 desensitizations in 23 patients, from evaluation to treatment. J Allergy Clin Immunol (2009) 124(6):1259-66. doi:10.1016/j.jaci.2009.09.009

36. Picard M, Castells MC. Re-visiting hypersensitivity reactions to taxanes: a comprehensive review. Clin Rev Allergy Immunol (2015) 49(2):177-91. doi:10.1007/s12016-014-8416-0

37. Giavina-Bianchi P, Galvão VR, Picard M, Caiado J, Castells MC. Basophil activation test is a relevant biomarker of the outcome of rapid desensitization in platinum compounds-allergy. J Allergy Clin Immunol Pract (2017) 5:728-36. doi:10.1016/j.jaip.2016.11.006

38. Lee C-W, Matulonis UA, Castells MC. Carboplatin hypersensitivity: a 6-h 12-step protocol effective in 35 desensitizations in patients with gynecological malignancies and mast cell/IgE-mediated reactions. Gynecol Oncol (2004) 95(2):370-6. doi:10.1016/j.ygyno.2004.08.002

39. Bavbek S, Ataman Ş, Akıncı A, Castells M. Rapid subcutaneous desensitization for the management of local and systemic hypersensitivity reactions to etanercept and adalimumab in 12 patients. J Allergy Clin Immunol Pract (2015) 3(4):629-32. doi:10.1016/j.jaip.2015.01.009

40. Nucera E, Schiavino D, Hohaus S, Leone G, Buonomo A, Lombardo C, et al. Desensitization to thalidomide in a patient with multiple myeloma. Clin Lymphoma Myeloma (2008) 8(3):176-8. doi:10.3816/CLM.2008.n.022

41. Breslow RG, Caiado J, Castells MC. Acetylsalicylic acid and montelukast block mast cell mediator-related symptoms during rapid desensitization. Ann Allergy Asthma Immunol (2009) 102(2):155-60. doi:10.1016/S1081-1206(10)60247-5

42. Clayton E, Madamba J, Kong X-T, Braskett M. Successful desensitization protocol for delayed cutaneous eruption to temozolomide. J Allergy Clin Immunol Pract (2014) 2(5):626-8. doi:10.1016/j.jaip.2014.03.016

43. Hesterberg PE, Banerji A, Oren E, Penson RT, Krasner CN, Seiden MV, et al. Risk stratification for desensitization of patients with carboplatin hypersensitivity: clinical presentation and management. J Allergy Clin Immunol (2009) 123(6):1262-7.e1. doi:10.1016/j.jaci.2009.02.042

44. Markman M, Kennedy A, Webster K, Elson P, Peterson G, Kulp B, et al. Clinical features of hypersensitivity reactions to carboplatin. JClin Oncol (1999) 17(4):1141. doi:10.1200/JCO.1999.17.4.1141
45. Polyzos A, Tsavaris N, Kosmas C, Arnaouti T, Kalahanis N, Tsigris C, et al. Hypersensitivity reactions to carboplatin administration are common but not always severe: a 10-year experience. Oncology (2001) 61(2):129-33. doi:10.1159/000055363

46. Moon DH, Lee JM, Noonan AM, Annunziata CM, Minasian L, Houston $\mathrm{N}$, et al. Deleterious BRCA1/2 mutation is an independent risk factor for carboplatin hypersensitivity reactions. Br J Cancer (2013) 109(4):1072-8. doi:10.1038/bjc.2013.389

47. Galvão VR, Phillips E, Giavina-Bianchi P, Castells MC. Carboplatin-allergic patients undergoing desensitization: prevalence and impact of the BRCA 1/2 mutation. J Allergy Clin Immunol Pract (2017) 5(3):816-8. doi:10.1016/j. jaip.2016.08.012

48. Thomas RR, Quinn MG, Schuler B, Grem JL. Hypersensitivity and idiosyncratic reactions to oxaliplatin. Cancer (2003) 97(9):2301-7. doi:10.1002/ cncr.11379

49. Maindrault-Goebel F, André T, Tournigand C, Louvet C, Perez-Staub $\mathrm{N}$, Zeghib N, et al. Allergic-type reactions to oxaliplatin: retrospective analysis of 42 patients. Eur J Cancer (2005) 41(15):2262-7. doi:10.1016/j. ejca.2005.06.021

50. Baldo BA. Adverse events to monoclonal antibodies used for cancer therapy: focus on hypersensitivity responses. Oncoimmunology (2013) 2(10):e26333. doi:10.4161/onci.26333

51. Caiado J, Venemalm L, Pereira-Santos MC, Costa L, Barbosa MP, Castells M. Carboplatin-, oxaliplatin-, and cisplatin-specific IgE: cross-reactivity and value in the diagnosis of carboplatin and oxaliplatin allergy. J Allergy Clin Immunol Pract (2013) 1(5):494-500. doi:10.1016/j.jaip.2013.06.002

52. Lee C, Gianos M, Klaustermeyer WB. Diagnosis and management of hypersensitivity reactions related to common cancer chemotherapy agents. Ann Allergy Asthma Immunol (2009) 102(3):179-87; quiz 187-9, 222. doi:10.1016/ S1081-1206(10)60078-6

53. Vasey PA, Jayson GC, Gordon A, Gabra H, Coleman R, Atkinson R, et al. Phase III randomized trial of docetaxel-carboplatin versus paclitaxel-carboplatin as first-line chemotherapy for ovarian carcinoma. J Natl Cancer Inst (2004) 96(22):1682-91. doi:10.1093/jnci/djh323

54. Squibb B-M. TAXOL ${ }^{\circledR}$ (Paclitaxel) Injection Prescribing Information. FDA, (2012).

55. Qiu S, Liu Z, Hou L, Li Y, Wang J, Wang H, et al. Complement activation associated with polysorbate 80 in beagle dogs. Int Immunopharmacol (2013) 15(1):144-9. doi:10.1016/j.intimp.2012.10.021

56. Weiszhár Z, Czúcz J, Révész C, Rosivall L, Szebeni J, Rozsnyay Z. Complement activation by polyethoxylated pharmaceutical surfactants: cremophor-EL, tween-80 and tween-20. Eur J Pharm Sci (2012) 45(4):492-8. doi:10.1016/j. ejps.2011.09.016

57. Hennenfent KL, Govindan R. Novel formulations of taxanes: a review. Old wine in a new bottle? Ann Oncol (2006) 17(5):735-49. doi:10.1093/annonc/mdj100

58. Sheeley DM, Merrill BM, Taylor LC. Characterization of monoclonal antibody glycosylation: comparison of expression systems and identification of terminal alpha-linked galactose. Anal Biochem (1997) 247(1):102-10. doi:10.1006/ abio.1997.2036

59. Chung CH, Mirakhur B, Chan E, Le QT, Berlin J, Morse M, et al. Cetuximabinduced anaphylaxis and IgE specific for galactose-alpha-1,3-galactose. $N$ Engl $J$ Med (2008) 358(11):1109-17. doi:10.1056/NEJMoa074943

60. Doessegger L, Banholzer ML. Clinical development methodology for infusion-related reactions with monoclonal antibodies. Clin Transl Immunology (2015) 4(7):e39. doi:10.1038/cti.2015.14

61. Luheshi GN. Cytokines and fever. Mechanisms and sites of action. Ann N Y Acad Sci (1998) 856:83-9. doi:10.1111/j.1749-6632.1998.tb08316.x

Conflict of Interest Statement: The author declares that the research was conducted in the absence of any commercial or financial relationships that could be construed as a potential conflict of interest.

Copyright $(02017$ Castells. This is an open-access article distributed under the terms of the Creative Commons Attribution License (CC BY). The use, distribution or reproduction in other forums is permitted, provided the original author(s) or licensor are credited and that the original publication in this journal is cited, in accordance with accepted academic practice. No use, distribution or reproduction is permitted which does not comply with these terms. 